\title{
Dependence of the lamellar thickness of an extended-chain single crystal of polyethylene on the degree of supercooling and the pressure. Faraday Transactions.
}

Citation for published version (APA):

Hikosaka, M., Okada, H., Toda, A., Rastogi, S., \& Keller, A. (1995). Dependence of the lamellar thickness of an extended-chain single crystal of polyethylene on the degree of supercooling and the pressure. Faraday Transactions. Journal of the Chemical Society, Faraday Transactions, 91(16), 2573-2579. https://doi.org/10.1039/ft9959102573

DOI:

10.1039/ft9959102573

Document status and date:

Published: 01/01/1995

\section{Document Version:}

Publisher's PDF, also known as Version of Record (includes final page, issue and volume numbers)

\section{Please check the document version of this publication:}

- A submitted manuscript is the version of the article upon submission and before peer-review. There can be important differences between the submitted version and the official published version of record. People interested in the research are advised to contact the author for the final version of the publication, or visit the DOI to the publisher's website.

- The final author version and the galley proof are versions of the publication after peer review.

- The final published version features the final layout of the paper including the volume, issue and page numbers.

Link to publication

\footnotetext{
General rights

- You may freely distribute the URL identifying the publication in the public portal. follow below link for the End User Agreement:

www.tue.nl/taverne

\section{Take down policy}

If you believe that this document breaches copyright please contact us at:

openaccess@tue.nl

providing details and we will investigate your claim.
}

Copyright and moral rights for the publications made accessible in the public portal are retained by the authors and/or other copyright owners and it is a condition of accessing publications that users recognise and abide by the legal requirements associated with these rights.

- Users may download and print one copy of any publication from the public portal for the purpose of private study or research.

- You may not further distribute the material or use it for any profit-making activity or commercial gain

If the publication is distributed under the terms of Article $25 \mathrm{fa}$ of the Dutch Copyright Act, indicated by the "Taverne" license above, please 


\title{
Dependence of the Lamellar Thickness of an Extended-chain Single Crystal of Polyethylene on the Degree of Supercooling and the Pressure
}

\author{
M. Hikosaka, ${ }^{*}$ H. Okada and A. Toda \\ Division of Material and Life Sciences, Faculty of Integrated Arts and Sciences, Hiroshima \\ University, Higashi-Hiroshima, 724 Japan
}

5. Rastogi

Department of Textile Technology, Indian Institute of Technology, New Delhi 110016, India

A. Keller

H. H. Wills Physics Laboratory, University of Bristol, Bristol, UK BS8 1TL

\begin{abstract}
The dependence of the lamellar thickness $(I)$ of an extended-chain single crystal (ECSC) of polyethylene (PE) crystallized at various pressures below or at the triple-point pressure $\left(P_{\text {tri }}=0.5 \mathrm{GPa}\right)$ on the degree of supercooling $(\Delta T)$ and the pressure have been studied. The value of $/$ increased with the decrease in $\Delta T$ [i.e. increase in the crystallization temperature $\left.\left(T_{c}\right)\right]$ at a fixed pressure, similar to the well known $\Delta T$ dependence of $/$ for a folded-chain single crystal (FCC). The observed maximum value of $l$, obtained at the lowest $\Delta T\left(I_{\max }\right)$ increased with increasing pressure and the crystal changed from FCC to an extended-chain crystal (ECC) at ca. $0.25 \mathrm{GPa}$. Application of the chain-sliding diffusion theory, previously proposed by one of the authors (M.H.) was found to explain well observed significant $\Delta T$ dependence of / and the pressure dependence of $I_{\max }$. It was proposed that the value of $/$ is determined by the cessation of lamellar thickening growth at the phase transition from metastable hexagonal to stable orthorhombic. The phase transition was also studied and it is suggested to be a nucleation-controlled process of the primary nucleus.
\end{abstract}

\section{Introduction}

The question: What determines the lamellar thickness of polymer crystals? is one of the most important unsolved problems in polymer science, because it is closely related to crystallization kinetics and the thickness strongly influences the physical properties of the solid, such as melting temperature, ${ }^{1}$ ferroelectric properties ${ }^{2}$ etc.

It is well known that $l$ for FCCs increases with crystallization temperature or annealing temperature. ${ }^{1}$ Hoffman et al. proposed a nucleation theory to explain the temperature dependence of $l$ for $\mathrm{FCCs}^{3}$ and Sadler proposed an entropic-controlled theory. ${ }^{4}$

Recently, we found that an ECSC of PE is formed via coupling of the well known lateral growth and the newly found lamellar thickening growth, ${ }^{5,6}$ as illustrated in Fig. 1. Note that the cross-section of an ESCS exhibits a tapered shape and so $l$ is defined as the maximum thickness at the centre.

It is found that $l$ increases linearly with time $(t)$, from which the lamellar thickening growth rate $(u)$ defined by $(\mathrm{d} l / \mathrm{d} t) / 2$ may be obtained. ${ }^{6}$ Thus, $l$ is given by

$$
l=l^{*}+2 u t
$$

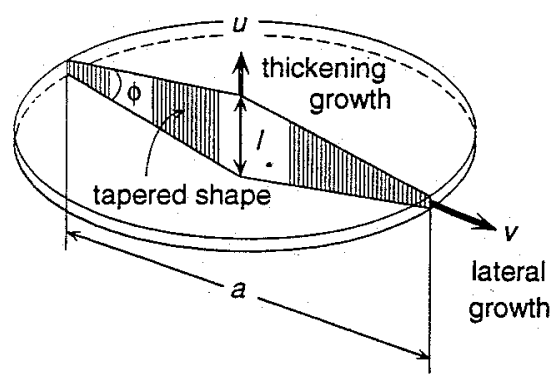

Fig, 1 Schematic diagram of an ECSC of PE. Two types of growth ate shown. Lateral size, $a$, is defined as the diameter of the ECSC. where $l^{*}$ is the value of $l$ for a critical nucleus. It was found that $u$ increases with increase in the degree of supercooling $(\Delta T)$ and hence we can derive the equation:

$$
u=C \exp (-D / \Delta T)
$$

where $C \approx 10^{2} \mathrm{~nm} \mathrm{~s}^{-1}$ and $D \approx 20 \mathrm{~K}^{7}$ Eqn. (2) is obtained by very careful observation of ECSCs crystallized at $0.4 \mathrm{GPa}$. Rough observations of $u$ for ECSCs crystallized at 0.3 and 0.5 $G P a$ showed that $D$ does not depend significantly on pressure, whereas $C$ seems to increase with pressure.

It was also found that the experimental formula for $u$ is very similar to that for the lateral growth rate $(v)$ of ECSCs,

$$
v=A \exp (-B / \Delta T)
$$

where $A \approx 10^{3} \mathrm{~nm} \mathrm{~s}^{-1}$ and $B \approx 23 \mathrm{~K}$ at $0.4 \mathrm{GPa}^{7}$ It was shown that $B$ does not depend on pressure, but that $A$ decreases slightly with increase in pressure. ${ }^{6}$ Therefore it was concluded that the following relations are applicable for the pressure and temperature studied

$$
u \propto v
$$

or

$$
D \approx B \approx 20 \mathrm{~K}
$$

Eqn. (4) and (5) are referred to throughout the present work. Eqn. (4) means that the cross-section of an ECSC does not change with growth.

We confirmed the important role of the mesophase, e.g. the hexagonal phase, in the formation of ECSCs where chainsliding is easy $^{6}$ as predicted by the chain-sliding diffusion theory previously presented by one of us. ${ }^{8}$ This theory predicted that $u$ for a hexagonal (hex) crystal $[u(h)]$ is much 


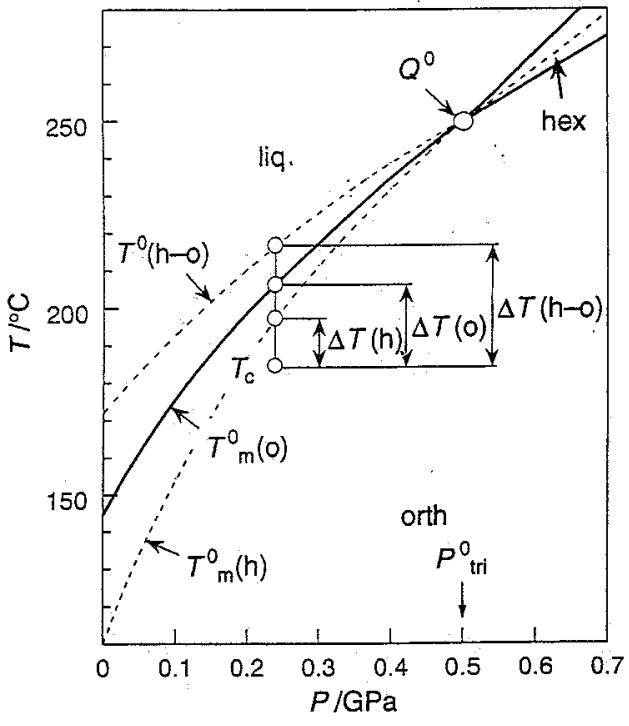

Fig. 2 Equilibrium pressure $(P)$-temperature $(T)$ phase diagram for $P E$ constructed from Fig. 7 in ref. $9 . Q^{\circ}$ indicates the triple point and $P_{\mathrm{tri}}$ is the triple-point pressure $\left(P_{\mathrm{tri}}=0.5 \mathrm{GPa}\right) . \Delta T(\mathrm{~h}), \Delta T(\mathrm{o})$ and $\Delta T(\mathrm{~h}-\mathrm{o})$ are defined in the text.

greater than that of an orthorhombic (orth) crystal $[u(0)]$

$$
u(\mathrm{~h}) \gg u(\mathrm{o})
$$

We constructed the equilibrium pressure-temperature $(P-T)$ phase diagram for PE shown in Fig. $2^{9}$ and found that the equilibrium triple-point pressure $\left(P_{\text {tri }}\right)$ is equal to $c a .0 .5$ $\mathrm{GPa}$. We also found that ECSCs can be formed even below the $P_{\text {tri }}$. In this case the hex phase is thermodynamically metastable. We found that the metastable hex crystal transforms into a stable orth crystal with growth after a lifetime $[t(\mathrm{~h}-\mathrm{o})]$. From this point onwards we were investigating the new concept of 'stability inversion with growth'.10

Recently, we found that the value of $l$ for an ECSC formed below the $P_{\text {tri }}$ increases with decrease in $\Delta T$ (or increase in $T)^{9}$ which is very similar to what happens with an FCC. ${ }^{1}$ This may be explained by a combination of the above results and considerations.

The purpose of this study is to show the temperature and pressure dependence of the lamellar thickness of an ECSC of PE formed below or at $P_{\text {tri }}$, to present a new theory to explain the results found and to confirm the theory by comparison with the observed results.

\section{Experimental}

The material used in this study was fully fractionated PE (NIST, SRM1483, $M_{\mathrm{w}}=32 \times 10^{3}, M_{\mathrm{w}} / M_{\mathrm{n}}=1.11$ ).

A $P-T$ phase diagram for PE was constructed, as shown in Fig. 2, by extrapolation from the equilibrium $P-T$ phase diagram in ref. 8. $T_{\mathrm{m}}^{0}(\mathrm{~h}), T_{\mathrm{m}}^{0}(\mathrm{o})$ and $T^{\mathrm{o}}(\mathrm{h}-\mathrm{o})$ are the equilibrium melting temperatures of a hex crystal and an orth crystal, and the hex-orth transition temperature, respectively, liq. indicates liquid phase and $Q^{\circ}$ the equilibrium triple point. $P_{\text {tri }}$ was $0.5 \mathrm{GPa}$. The solid curves indicate the equilibrium transition, while the dotted lines represent the metastable states.

Three types of degree of supercooling $(\Delta T)$ were distinguished in this study: $:^{9} \Delta T(\mathrm{~h})$ for a hexagonal crystal, $\Delta T(\mathrm{o})$ for an orthorhombic crystal and $\Delta T(\mathrm{~h}-\mathrm{o})$ for the hex to orth transformation.

$$
\begin{gathered}
\Delta T(\mathrm{~h}) \equiv T_{\mathrm{m}}^{0}(\mathrm{~h})-T_{\mathrm{c}} ; \quad \Delta T(\mathrm{o}) \equiv T_{\mathrm{m}}^{0}(\mathrm{o})-T_{\mathrm{c}} ; \\
\Delta T(\mathrm{~h}-\mathrm{o}) \equiv T^{0}(\mathrm{~h}-\mathrm{o})-T_{\mathrm{c}}
\end{gathered}
$$

PE was crystallized isothermally from the melt under given pressure from 1 atm up to $P_{\text {tri }}=0.5 \mathrm{GPa}$ (Fig. 2) Experimental details were given in ref. 8 . The crystallizatio behaviour and crystal structures were observed directly by both polarizing optical microscopy and $\mathrm{X}$-ray diffraction.

The lamellar thickness of a single crystal was measured by using transmission electron microscopy. $l$ shows a wide dis tribution, even on a sample crystallized under identical condi tions, i.e. at a given set of $\Delta T(\mathrm{~h})$ and $P$. Note that, in this study, the observed value of $l$ was defined as the maximun one obtained under the given conditions. In order to obtain the maximum $l$, the crystallization time was allowed to be sufficiently long; further details are given in the next section.

\section{Effect of $\Delta T(h)$ on $l$}

\section{Results}

A wide distribution in the values of $l$ was observed on many single crystals within a sample crystallized under given condi tions. However, there exists a maximum lamellar thickness for a given $\Delta T(\mathrm{~h})$ and $P$ and in this study we define the maximum lamellar thickness as the largest $l$ that could be observed. It was confirmed that the maximum lamellar thickness was obtained usually on single crystals which have transformed from hex to orth. The physical interpretation of the close connection between the maximum lamellar thick ness and the phase transition will be given in Sections IV and $\mathrm{V}$.

Fig. 3 shows the variation of $l$ with $\Delta T(\mathrm{~h})$ observed at 0.3 0.4 and $0.5 \mathrm{GPa}$ for $\Delta T(\mathrm{~h})<14 \mathrm{~K}$. It can be seen that increases significantly with decrease in $\Delta T(\mathrm{~h})$ (i.e. increase in $T_{c}$ ) for $\Delta T(\mathrm{~h})$ less than a few $\mathrm{K}$. This is very similar to the well known $\Delta T$ dependence of $l$ for folded-chain single crys tals.

\section{Effect of Pressure on $l_{\max }$}

We could define an observed maximum value of $l\left(l_{\max }\right)$ for $a$ given pressure at the lowest observed $\Delta T(h)$. In Fig. 3 the $l_{\text {ma }}$ values for $0.3,0.4$ and $0.5 \mathrm{GPa}$ are indicated by arrows.

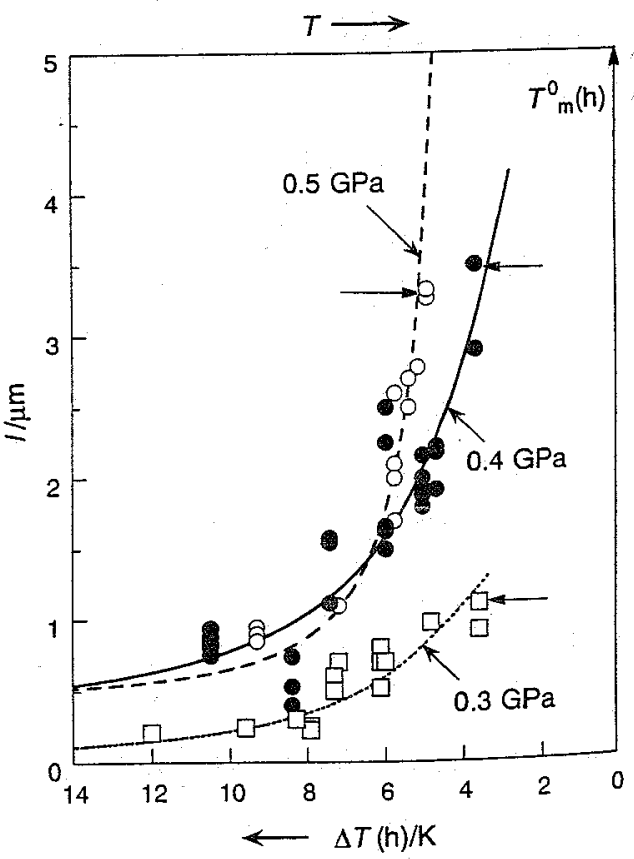

Fig. $3 l$ vs. $\Delta T(\mathrm{~h})$ or $T$ at indicated pressures. Points are observed values, curves are calculated as described in the text. Arrows indicate the observed maximum lamellar thickness $\left(l_{\max }\right)$ for $0.3,0.4$ and 05 GPa. 
Fig. 3 shows significant pressure dependence of the $l \mathrm{vs}$. $\Delta T(\mathrm{~h})$ curve with an upward shift with increasing pressure, especially marked at low $\Delta T(\mathrm{~h})$. In order to show this point nuch more clearly, we plotted $l_{\max }$ vs. pressure in Fig. 4. $l_{\max }$ increased with increasing pressure, tending to infinity at $P_{\text {tri }}$. The smooth curve is a theoretical one constructed from $l_{\text {th }}$ at $\Delta T(\mathrm{~h})=4 \mathrm{~K}$ for $P>0.3 \mathrm{GPa}$, see Section V. Below $0.3 \mathrm{GPa}$ the curve is extrapolated.

As the extended chain length of PE used in this study is 0.3 am, the crystals that crystallized below ca. $0.25 \mathrm{GPa}$ are FCCs, while those that crystallized above $c a .0 .25 \mathrm{GPa}$ are ECCs. Thus it is concluded that crystals of PE passed from the FCC to the ECC regime on increasing the pressure to $c a$. $0.25 \mathrm{GPa}$. Note that we will be able to obtain very thick ECCs or ECSCs (thicker than $10 \mu \mathrm{m}$ ) if we crystallize PE above the triple-point pressure.

\section{Evidence for the Metastable Hexagonal-Orthorhombic Transition}

We have stressed in previous work that, below the triplepoint pressure, a metastable hex crystal was first formed and then this was transformed into a stable orth crystal after some waiting time and that the lateral growth actually stops at the hex-orth transition. ${ }^{7-10}$

Fig. 5 shows typical evidence of the metastability of the hex crystal isothermally crystallized at $P=0.3 \mathrm{GPa}<P_{\text {tri }}$. The lower and upper crystals in Fig. 5(a) are hex and orth crystals, respectively. A simple optical identification of a hex crystal and an orth one has already been reported in ref. 7. A hex crystal can be identified from its optical image of a uniformly bright streak, whereas an orth crystal gives a blotchy, somewhat distorted, streak.

When the temperature is increased, the hex crystal begins to melt and diminishes, while the orth one remains, as shown in Fig. 5(b). This clearly shows that the melting temperature of the hex crystal $\left[T_{\mathrm{m}}^{\mathrm{O}}(\mathrm{h})\right]$ is lower than that of the orth crystal $\left[T_{\mathrm{m}}^{0}(\mathrm{o})\right]$. Therefore the hex crystal is metastable and the orth crystal is the stable form.

Fig. 6 shows the instantaneous hex-orth transition observed directly by polarizing optical microscopy and recorded by video camera. A hexagonal crystal grew contin-

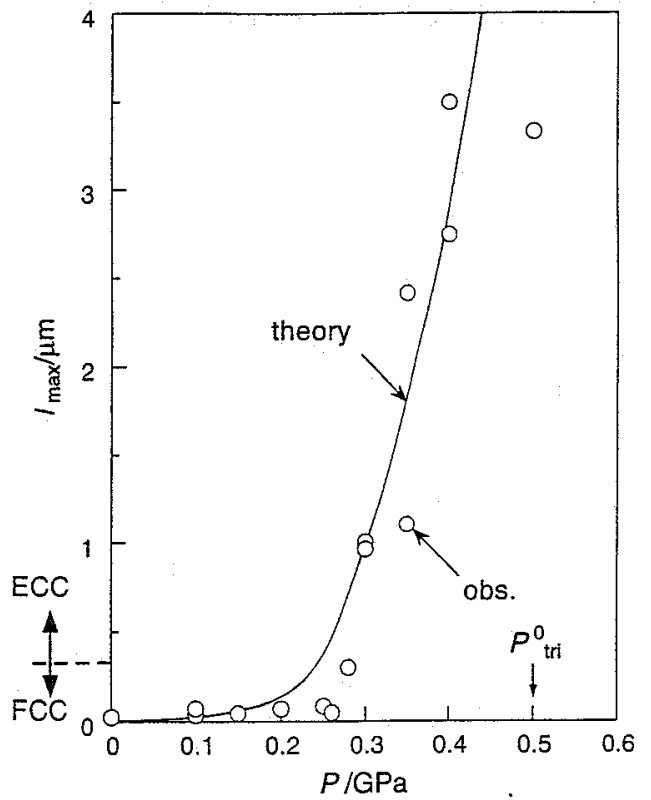

fig. $4 l_{\max } v s . P$. Points are observed values, curves are calculated as described in the text. The broken line at $0.3 \mu \mathrm{m}$ indicates the extended chain length of the material. (a)

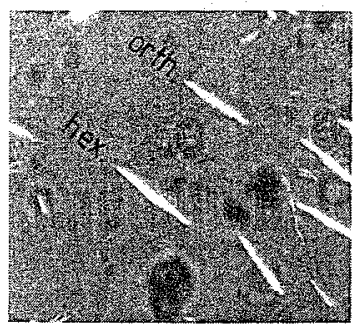

(b)

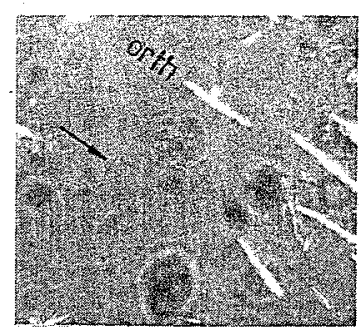

Fig. 5 Direct evidence of the metastability of the hexagonal crystal crystallized at $P=0.3 \mathrm{GPa}<P_{\text {tri }}$ observed by polarizing optical microscopy and automatic roll-film camera system. (a) Both hex and orth crystals are coexisting and growing at $T<T_{\mathrm{m}}^{0}(\mathrm{~h})$; (b) only the hex crytal has melted and diminished (indicated by arrow) at $T_{\mathrm{m}}^{\mathrm{0}}(\mathrm{h})<T<T^{0}(\mathrm{~h}-\mathrm{O})$.

uously within some time period after generation and then was transformed into an orthorhombic crystal within $c a .40$ $\mathrm{ms}$ (which is the limit of time-resolution of our video system). The time period (which we will call 'life-time' of the hexagonal crystal), ca. $98 \mathrm{~min}$ in this case, depended strongly on $\Delta T(\mathrm{~h})$, as will be reported in the next section.

At the same time, the lateral growth stopped, as shown in Fig. 7. Fig. 7 shows that the lateral size of an isolated single crystal $(a)$ increases linearly with time $(t)$ when the crystal is in the metastable hex phase but then remains virtually constant after the hex-orth transition.

(a)

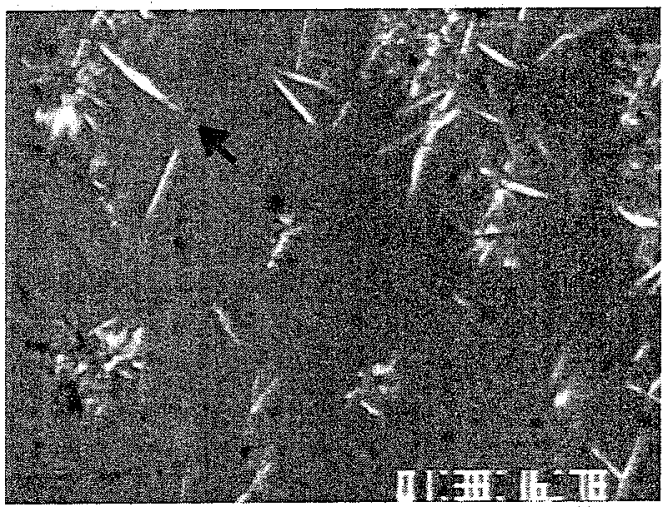

(b)

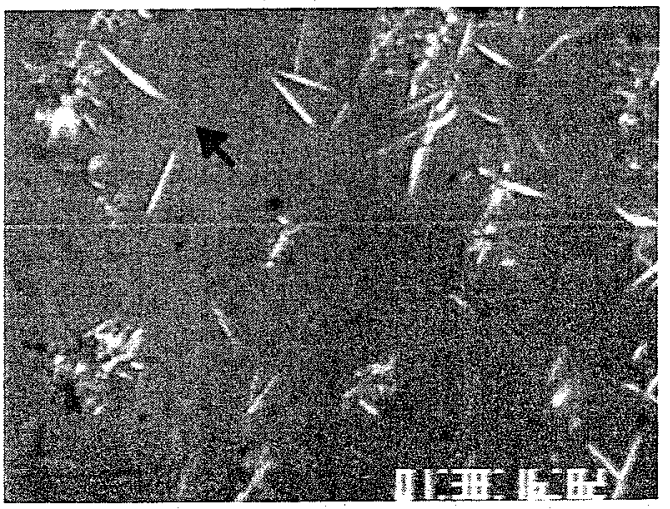

Fig. 6 Direct evidence of the instantaneous hex-orth transition observed on a single crystal (indicated by arrow) using polarizing optical microscopy and a video camera system. $P=0.4 \mathrm{GPa}$, $\Delta T(\mathrm{~h})=3.5 \mathrm{~K}:$ (a) just before and (b) just after the transition which took only $40 \mathrm{~ms}$. The transition occurred after an induction time which we call the lifetime of the hex crystal, $t(h-o)=98 \mathrm{~min}$. 


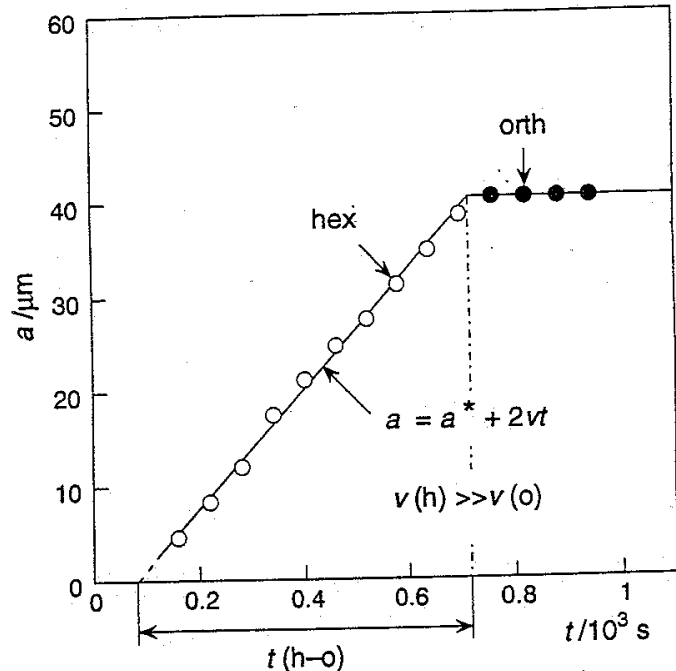

Fig 7 Lateral size $(a) v$ s. time $(t)$. Cessation of lateral growth of an ECSC at the hex-orth phase transition is shown. Definition of the lifetime of the metastable hex crystal is illustrated on the horizontal axis. $P=0.5 \mathrm{GPa}, \Delta T(\mathrm{~h})=6.2 \mathrm{~K}$.

\section{Dependence of the Lifetime of the Metastable Hex Crystal on} $\Delta T(h)$

Here we will define the lifetime, $t(\mathrm{~h}-0)$, of the metastable hex crystal as the time period from generation of a hex crystal to the hex-orth transiormation as shown in Fig. 7. The observed $t(\mathrm{~h}-\mathrm{o})$ at various pressures $(0.3,0.4$ and $0.5 \mathrm{GPa})$ was plotted vs. $\Delta T(\mathrm{~h})$ in Fig. 8. The curves are theoretical ones that will be explained in Section V.

Fig. 8 shows that $t(\mathrm{~h}-0)$ increases with decrease in $\Delta T(\mathrm{~h})$ (i.e. with decrease in $T_{\mathrm{c}}$ ). Note that $t(\mathrm{~h}-\mathrm{O})$ at all pressures tends to infinity at $\Delta T(\mathrm{~h})=0$; this will be discussed in Section IV and V.

\section{Theory of Lamellar-thickness-determining Mechanism}

Here the observed $\Delta T$ dependence and pressure dependence of the lamellar thickness will be explained theoretically by formulating $l$. The theory will be constructed by combining

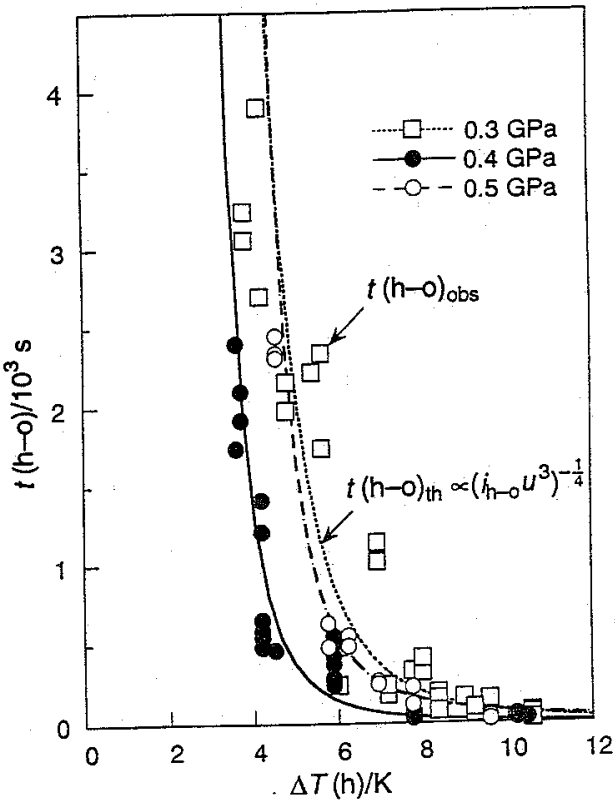

Fig. $8 t(\mathrm{~h}-\mathrm{o}) v s . \Delta T(\mathrm{~h})$ at $P=0.3,0.4$ and $0.5 \mathrm{GPa}$. The points are

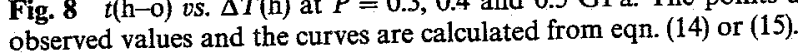

the idea that all ESCS which start from metastable hex crystals transform into stable ortho crystals after some lifetime of the hexagonal crystal and that the lamellar thickening growth nearly stops at the hex-orth transition. This is illustrated schematically in Fig. 9 which shows that the actual lamellar thickness of an orth crystal corresponds closely to that at the hex-orth transition, $l[t(\mathrm{~h}-\mathrm{o})]$.

Thus we will be able to formulate the lamellar thickness as a function of the lamellar thickening growth rate of the hexagonal crystal $u(\mathrm{~h})$ and the lifetime $[t(\mathrm{~h}-\mathrm{o})]$. The life-time will be also formulated as a function of the nucleation rate of an orth primary nucleus $\left(i_{\mathrm{h}-\mathrm{o}}\right)$ within the hex crystal and the volume of the 'mother' orth crystal $(\Phi)$.

\section{Cessation of Thickening Growth at the Hex-Orth Phase Transition}

First, we will formulate the lamellar thickness as a function of lamellar thickening growth rate of the hex crystal $u(\mathrm{~h})$ and the lifetime $[t(\mathrm{~h}-\mathrm{o})]$. Combining the hex-orth transition below $P_{\text {tri }}$ and the marked decrease in $u$ with the transition [eqn. (6)] gives us an indication that the lamellar thickening growth will nearly stop at the hex-orth transition. Thus we have an approximated formula for $l$ of an orth crystal,

$$
l \approx l^{*}+2 u t(\mathrm{~h}-\mathrm{o})
$$

It is well known that $l^{*}$ becomes infinite at $\Delta T(\mathrm{~h}) \approx 0 \mathrm{~K}$, therefore $l$ will become infinite. For $\Delta T(\mathrm{~h})>$ a few $\mathrm{K}$, on the other hand, $l^{*}$ can be neglected compared with $l$, in the case of an ECSC. Therefore it is approximated that

$$
\begin{array}{ll}
l \approx \infty ; & \text { for } \Delta T(\mathrm{~h}) \approx 0 \mathrm{~K} \\
l \approx 2 u t(\mathrm{~h}-\mathrm{o}) ; & \text { for } \Delta T(\mathrm{~h})>\mathrm{a} \text { few } \mathrm{K}
\end{array}
$$

Here we will assume $u \propto v$ [eqn. (4)] and $D \approx B \approx 20 \mathrm{~K}$ [eqn (5)] for the present pressure and temperature ranges for reasons already mentioned in the Introduction.

\section{Nucleation Rate $\left(i_{\mathrm{h}-0}\right)$ of the Primary Nucleus in the Hex-Orth Phase Transition}

Next we will formulate the nucleation rate $i_{\mathrm{h}-0}$. It will be shown in Section $\mathrm{V}$ that the hex-orth transition is mainly

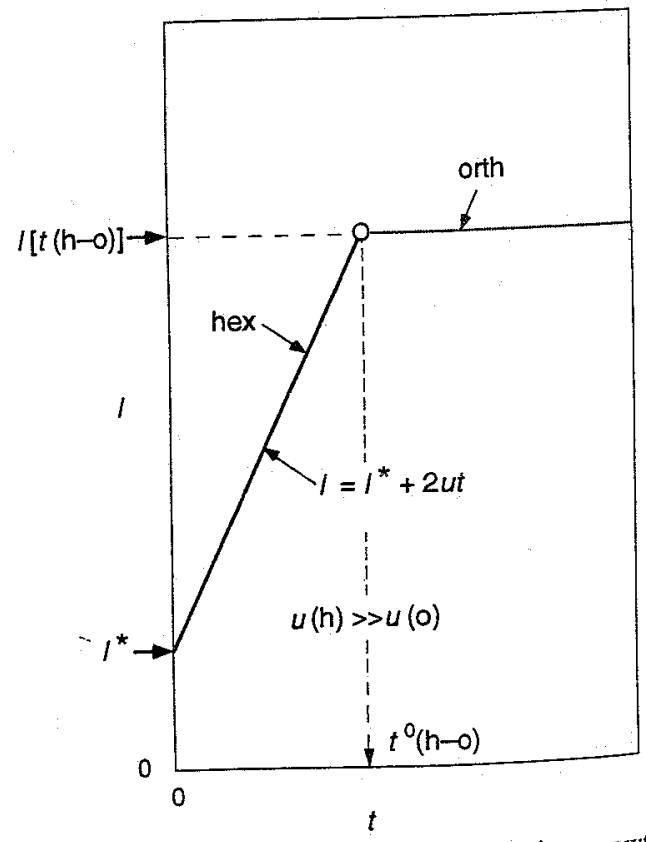

Fig. 9 Schematic illustration of lamellar thickening growth of an ECSC at $P<P_{\text {ti }}$. Cessation of the lamellar thickening growth at the hex-orth transition is shown. 


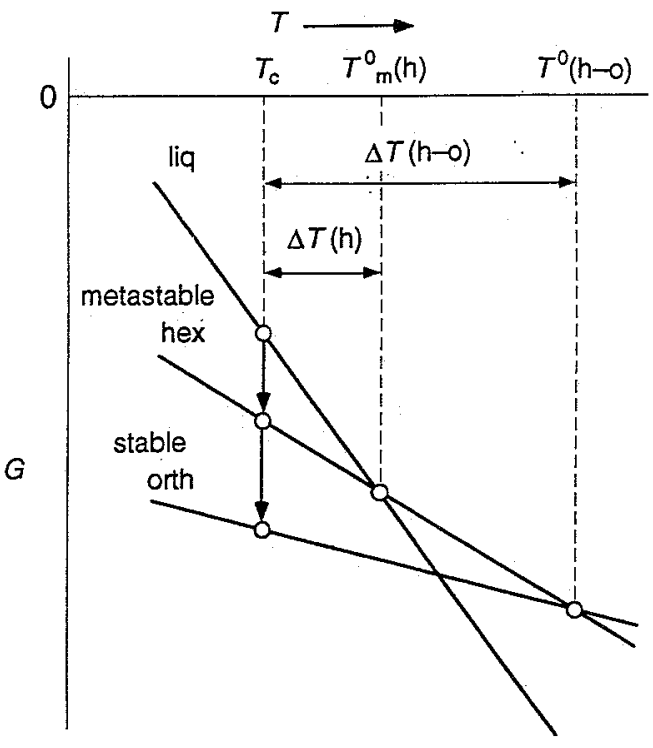

Fig. 10 Schematic illustration of the Gibbs energy of the liquid and metastable hexagonal and stable orthorhombic phase of PE vs. T at P $<<P_{\mathrm{tri}}$. Definitions of $\Delta T(\mathrm{~h})$ and $\Delta T(\mathrm{~h}-\mathrm{o})$ are shown again (Fig. 2). The typical step-wise change corresponding to Ostwald's step rule is shown by arrows.

controlled by a nucleation process of the hex primary pucleus. Therefore the nucleation rate $\left(i_{\mathrm{h}-\mathrm{o}}\right)$ of an orth primary nucleus within the 'mother' hex crystal is directly related to the life-time $t(\mathrm{~h}-\mathrm{o})$.

The hex-orth transition below $P_{\text {tri }}$ can be easily understood from the Gibbs energy $(G)$-temperature $(T)$ phase diagram shown in Fig. 10 and the so-called Ostwald's step rule. Fig. 10 indicates two types of $\Delta T$ related to our problem as already explained for Fig. 2. We have discussed the nature of the transformation in detail using the Gibbs-Thomson equation. ${ }^{10}$

It is obvious that the hex-orth transition is a first-order phase transition, therefore the transition must be controlled by a primary nucieation process. The observed fact that the hex-orth transition occurred instantaneously after a life-time $4(\mathrm{~h}-\mathrm{o})$ suggests that the transformation is a single nucleation process, so $t(\mathrm{~h}-\mathrm{o})$ is considered to be an observed induction time in the nucleation process.

Therefore we will assume that the hex-orth transition is a single nucleation process of the primary nucleus. Fig. 11 shows a schematic model of an orth primary nucleus (three-

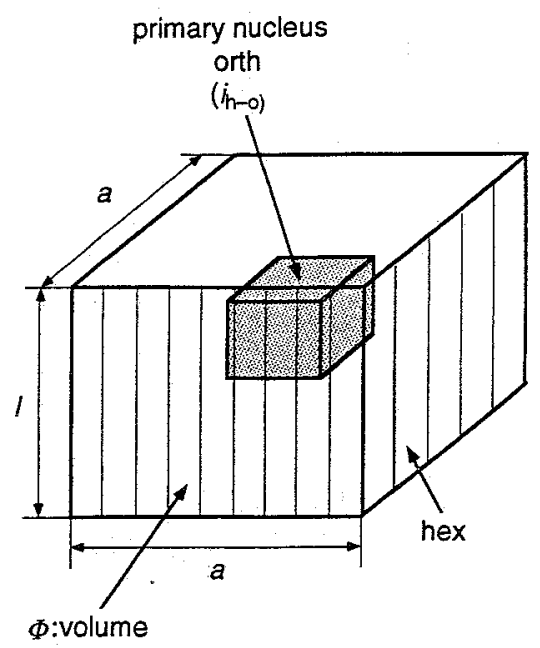

g. 11 Simple model of a primary nucleus (i.e. three-dimensional ucleus) in the metastable hex-stable orth transition. dimensional nucleus) nucleated within a metastable hex single crystal. Here, the crystal is simplified to be a tetragonal prism with lateral size, $a \times a$, and thickness, $l$. Of course the size of the nucleus for the transformation should be bigger than that of the critical nucleus.

It is well known that the nucleation rate $\left(i_{\mathrm{h}-\mathrm{o}}\right)$ of a homogeneous primary nucleus is a function of $\Delta T(\mathrm{~h}-0)$ and is given by the relation

$$
i_{\mathrm{h} \rightarrow \mathrm{o}}=i_{\mathrm{h} \rightarrow \mathrm{o}}[\Delta T(\mathrm{~h}-\mathrm{o})]=q \exp \left[-r / \Delta T^{2}(\mathrm{~h}-\mathrm{o})\right]
$$

where $q$ and $r$ are constants. It is given in the nucleation theory that

$$
r \propto \sigma^{2} \sigma_{\mathrm{e}} / \Delta h^{2}
$$

where $\sigma$ and $\sigma_{\mathrm{e}}$ are the side- and end-surface energies between the hex and orth phases and $\Delta h$ is the enthalpy of the hexorth transition.

\section{Lifetime, $t(\mathbf{h}-\mathbf{0})$}

We will now formulate the $t(\mathrm{~h}-\mathrm{o})$ of the hex crystal. The lifetime is equal to an induction time of a primary nucleus as illustrated in Fig. 11. It is obvious that $t(\mathrm{~h}-\mathrm{o})$ is proportional to both the inverse of $i_{\mathrm{h} \rightarrow \mathrm{o}}$ and the inverse of the volume of the single crystal $(\Phi)$

$$
t(\mathrm{~h}-\mathrm{o}) \propto\left(i_{\mathrm{h}-\mathrm{o}} \Phi\right)^{-1}
$$

$\Phi$ at $t=t(\mathrm{~h}-\mathrm{o})$ is given by eqn. (A5) in the Appendix

$$
\Phi \propto v^{2} u t^{3}(\mathrm{~h}-0) \approx u^{3} t^{3}(\mathrm{~h}-0) ; \quad \text { for } \Delta T(\mathrm{~h})>\text { a few } \mathrm{K}
$$

where the relation $v \propto u$ [eqn. (4)] is used. Combining eqn. (12) and (13) gives

$$
t(\mathrm{~h}-\mathrm{o}) \propto\left(i_{\mathrm{h}-0} u^{3}\right)^{-1 / 4} ; \text { for } \Delta T(\mathrm{~h})>\text { a few } \mathrm{K}
$$

Combination of eqn. (2) and (10) leads to another representation

$$
\begin{array}{r}
t(\mathrm{~h}-\mathrm{o}) \propto\left\{\exp \left[-r / \Delta T^{2}(\mathrm{~h}-\mathrm{o})\right] \exp [-3 D / \Delta T(\mathrm{~h})]\right\}^{-1 / 4} ; \\
\text { for } \Delta T(\mathrm{~h})>\text { a few } \mathrm{K}
\end{array}
$$

Eqn. (14) can be rewritten:

$$
i_{\mathrm{h} \rightarrow \mathrm{o}} \propto t^{-4}(\mathrm{~h}-\mathrm{o}) u^{-3} ; \text { for } \Delta T(\mathrm{~h})>\text { a few } \mathrm{K}
$$

\section{Formulation of $l$}

Lastly, we will formulate $l$ as a function of $\Delta T(\mathrm{~h}-\mathrm{o})$ and $\Delta T(\mathrm{~h})$. Combination of eqn. (9) and (14) or (15) gives

$$
l \approx \infty ; \text { for } \Delta T(\mathrm{~h}) \approx 0 \mathrm{~K}
$$

and

$$
l \approx 2 u t(\mathrm{~h}-\mathrm{o}) \propto\left(i_{\mathrm{h}-\mathrm{o}}^{-1} u\right)^{1 / 4} ; \text { for } \Delta T(\mathrm{~h})>\text { a few } \mathrm{K}
$$

i.e.

$$
\begin{array}{r}
l \propto\left\{\exp \left[r / \Delta T^{2}(\mathrm{~h}-\mathrm{o})\right] \exp [-D / \Delta T(\mathrm{~h})]\right\}^{1 / 4} \\
\text { for } \Delta T(\mathrm{~h})>\mathrm{a} \text { few } \mathrm{K}
\end{array}
$$

Note that eqn. (18) shows that $l$ is not only a function of $\Delta T(\mathrm{~h})$ but also of $\Delta T(\mathrm{~h}-\mathrm{o})$. It includes two opposing processes, the first term is an increasing function of crystallization temperature, whilst the latter is a decreasing one.

\section{Comparison with Experimental Results}

Nucleation Rate in the Hex-Orth Transition, $i(h-0)$

The observed nucleation rate, $i_{\mathrm{h}-\mathrm{o}}$, was obtained from the observed life-time $t_{\mathrm{obs}}(\mathrm{h}-\mathrm{o})$ shown in Fig. 8 using eqn. 16. $i_{\mathrm{h} \rightarrow \mathrm{o}}$ 


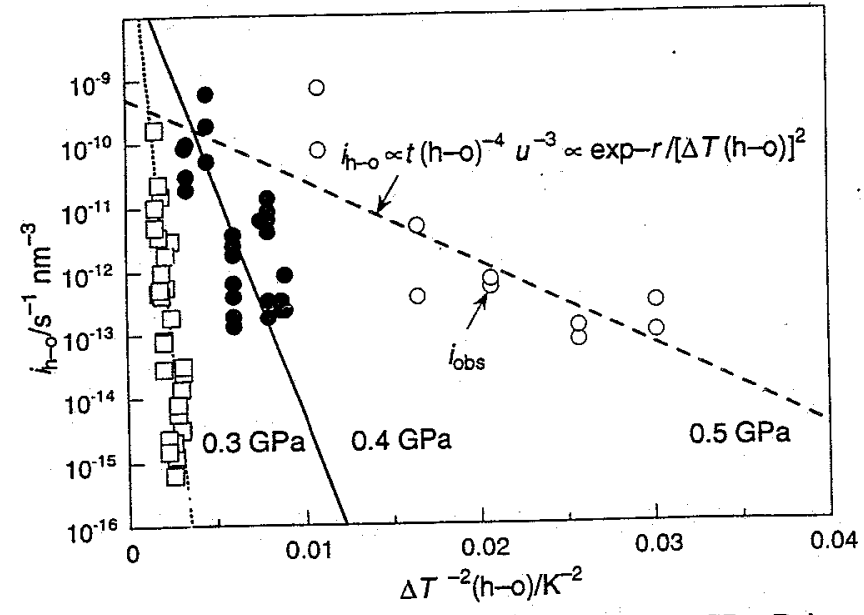

Fig. $12 \log [i(\mathrm{~h}-\mathrm{o})]$ vs. $\Delta T^{-2}(\mathrm{~h}-\mathrm{o})$ at $0.3,0.4$ and $0.5 \mathrm{GPa}$. Points are observed values and the straight lines are calculated using eqn. (16). The linear relations suggest that the hex-orth transition is a primary-nucleation controlled one.

was plotted vs. $1 / \Delta T^{2}(\mathrm{~h}-0)$ for $P=0.3,0.4$ and $0.5 \mathrm{GPa}$ in Fig. 12. This showed that $i_{\mathrm{h} \rightarrow \mathrm{o}}$ vs. $1 / \Delta T^{2}(\mathrm{~h}-\mathrm{o})$ gives fairly good straight lines, which suggests that the hex-orth transition is a single primary nucleation controlled process.

Note, also, that the slope $[r$ in eqn. (10)] decreases with increase in pressure. $\log (-r)$ is plotted vs. pressure in Fig. 13, from which we derive an experimental formula

$$
r \propto \exp (-w P)
$$

where $w$ is a constant, $w=16 \mathrm{GPa}^{-1}$.

This result suggests that the activation energy of the critical primary nucleus, which is proportional to $r=\sigma^{2} \sigma_{\mathrm{e}} / \Delta h^{2}$ decreases very swiftly with increase in pressure. The reason for this is unknown and poses an interesting new question.

Thus we can conclude that the hex-orth transition below $P_{\mathrm{tri}}$ is mainly controlled by the nucleation process of the primary nucleus.

\section{Dependence of $l$ on $\Delta T(\mathrm{~h})$ or $T_{\mathrm{c}}$}

Fig. 14 shows a typical $\Delta T(\mathrm{~h})$ or $T_{\mathrm{c}}$ dependence of $l$ for 0.4 $\mathrm{GPa}$. The points represent $l_{\mathrm{obs}}$ and the continuous curve is theoretical, calculated using eqn. (17) or (18). $l_{\mathrm{th}}$ contains two opposing factors, $i_{\mathrm{h} \rightarrow \mathrm{o}}^{-1}$ and $u$, which are also shown in Fig. 14. $i_{h \rightarrow 0}^{-1}$ is an increasing function of crystallization temperature, whilst $u$ is a decreasing one. In the calculation, the straight line of $i_{\mathrm{h} \rightarrow \mathrm{o}}$ in Fig. 12 was used. Observed $u$ was used for 0.4 $\mathrm{GPa}^{6}{ }^{6} l_{\mathrm{h}}$ was calculated for $\Delta T(\mathrm{~h})>3 \mathrm{~K}$. Below $3 \mathrm{~K}$ it is difficult to calculate because $i_{\mathrm{h}-\mathrm{a}}^{-1}$ tends to infinity as $\Delta T(\mathrm{~h})$ approaches $0 \mathrm{~K}$.

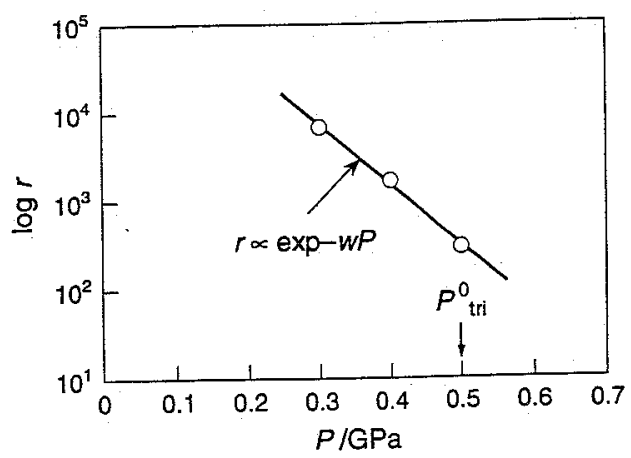

Fig. $13 \log (r)$ vs. $P$ obtained from the slope of $\log [i(\mathrm{~h}-\mathrm{o})]$ vs. $\Delta T^{-2}(\mathrm{~h}-\mathrm{o})$ shown in Fig. 12.

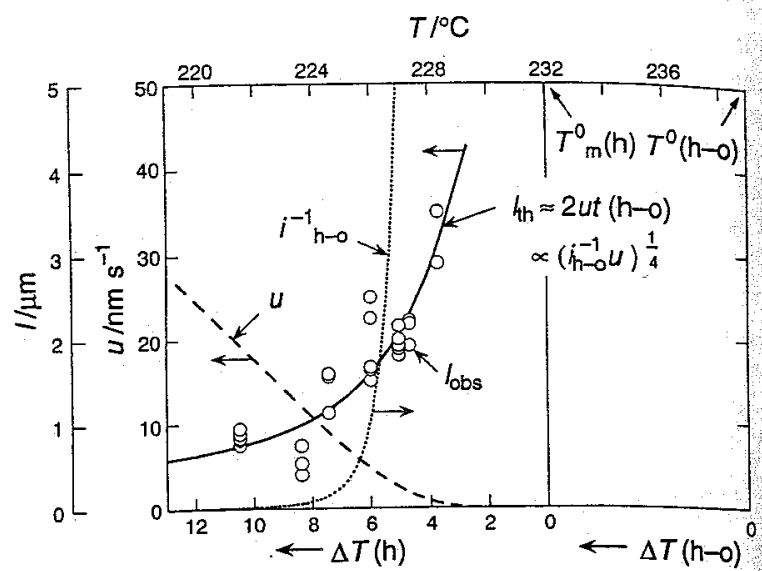

Fig. 14 Typical $l$ vs. $\Delta T(\mathrm{~h})$ at $P=0.4 \mathrm{GPa}<P_{\text {tri }}$. Points are observed values and the curves are calculated: $l_{\text {th }}$ from eqn. (17) or (18) and $u$ from eqn. (2) and $i(\mathrm{~h}-\mathrm{o})$ (given in Fig. 12).

$l_{\text {th }}$ increases with decrease in $\Delta T(\mathrm{~h})$ or increase in $T_{c}$, for the range $\Delta T(\mathrm{~h})>3 \mathrm{~K}$. This well explains the trend in the values of $l_{\text {obs }}$. It is shown that $l_{\text {th }}$ is controlled by both $i_{\mathrm{h} \rightarrow 0}[\Delta T(\mathrm{~h}-\mathrm{o})]$ and $u[\Delta T(\mathrm{~h})]$; therefore it is concluded that $l_{\mathrm{hh}}$ is a function of both $\Delta T(\mathrm{~h})$ and $\Delta T(\mathrm{~h}-\mathrm{o})$.

The dotted and broken curves in Fig. 3 indicate the values of $l_{\text {th }}$ for the two further pressures 0.3 and $0.5 \mathrm{GPa}$, respec tively. Again it is confirmed that $l_{\text {th }}$ increases with decrease in $\Delta T(\mathrm{~h})$ or increase in $T_{\mathrm{c}}$, for the range $\Delta T(\mathrm{~h})>3 \mathrm{~K}$. This again agrees well with the values of $l_{\text {obs }}$. They are calculated by a similar procedure to that shown above. As for $u$ for 0.3 and $0.5 \mathrm{GPa}$, we took $u$ as proportional to that observed at $0.4 \mathrm{GPa}$ because we have shown in the Introduction that the relations, $u \propto v$ or $D \approx B \approx 20 \mathrm{~K}$ are applicable at any pressure.

Thus we reach the most important conclusion, that the lamellar thickness is determined by apparent stoppage of lamellar thickening growth through the phase transition from metastable hex to the stable orth.

\section{$P$ Dependence of $\boldsymbol{l}$}

We have shown in Section IV that $l$ is controlled by two opposing factors, $i_{\mathrm{h}-\mathrm{o}}[\Delta T(\mathrm{~h}-\mathrm{o})]$ and $u[\Delta T(\mathrm{~h})]$. The next question is what determines the pressure dependence of 1 as shown in Fig. 3 or 4 . Fig. 3 shows that $l$ vs. $\Delta T(\mathrm{~h})$ shifts upward with increase in pressure and Fig. 4 shows that $l_{\text {max }}$ increases with increase in pressure.

In this study it is assumed that $D$ in the formula for $u$ [eqn (2)] does not depend significantly on pressure, therefore $u$ wil not play an important role in the present problem. $i_{\mathrm{h} \rightarrow 0}^{-1}$ is, on the other hand, not a function of $\Delta T(h)$ but a function of the other hand, not a function of $\Delta T(\mathrm{~h})$ but a function infinite at $\Delta T(\mathrm{~h}-\mathrm{o})=0$, as shown in Fig. 14 .

It is obvious that the difference between $\Delta T(\mathrm{~h}-\mathrm{O})$ and $\Delta T(\mathrm{~h})$ decreases with increase in pressure, as shown in Fig. 2 and the difference becomes zero at $P=0.5 \mathrm{GPa}$. Therefore, $\Delta T(\mathrm{~h}-\mathrm{o})$ at a constant $\Delta T(\mathrm{~h})$ decreases with increase in pressure, which will result in an increase in $i_{\mathrm{h} \rightarrow 0}^{-1}$. As $l$ is proportional to $\left(i_{\mathrm{h}-\mathrm{a}}^{-1} u\right)^{1 / 4}$, the above increase in $i_{\mathrm{h} \rightarrow 0}^{-1}$ will result in an increase in $l$ at $\Delta T(\mathrm{~h})$ or in $l_{\max }$ with increase of pressure. Thus it is concluded that the increase in $l$ or in $l$ at Thus it is concluded that the increase in $l_{\max }$, or ine in $\Delta T(\mathrm{~h})$, with pressure is mainly determined by the decteas

\section{Conclusions}

1. For an ECSC crystallized below or at $P_{\mathrm{tri}}(a)$ the lamel lar thickness increased with the degree of super cooling of the 
crystal, which is similar to the behaviour of FCCs; $(b)$ the observable maximum, $l_{\max }$, at a given pressure increased with increase in pressure, as a result of which a crystal passed over from the FCC to the ECC regime at $0.25 \mathrm{GPa}$ when the crystallization pressure was increased.

2. The metastable hex to stable orth phase transition was observed. The life-time of the metastable hexagonal crystal $[t(h-0)]$ was determined for the first time suggesting that the hex-orth transition is mainly controlled by the process of primary nucleation (i.e. three-dimensional nucleation). The observed nucleation rate $\left(i_{\mathrm{h}-\mathrm{o}}\right)$ obeyed the relation, $i_{\mathrm{h} \rightarrow \mathrm{o}}=q$ $\exp \left[-r / \Delta T^{2}(\mathrm{~h}-\mathrm{o})\right]$. It is found that the activation energy of the critical primary nucleus decreases with increase in pressure.

3. A new theory was presented to show that the lamellar thickness of an ECSC is determined by cessation of the thickening growth at the hex-orth phase transition. The formula derived for the lamellar thickness shows that the lamellar thickness is controlled by two opposing factors, the nucleation rate of the primary nucleus and the lamellar thickening growth rate. This well explained the observed $\Delta T(\mathrm{~h})$ and pressure dependence of $l$, confirming the validity of the theory.

We are grateful to Prof. H. Ohigashi for helpful discussions and to Mr. K. Amano for his experiment assistance. This work was partly supported by Grant-in-Aid for Scientific Research C No. 6651038 and B No. 07455386 from Ministry of Education, Japan.

\section{Appendix}

\section{Volume of a Single Crystal $(\Phi)$}

In order to formulate the life-time $t(\mathrm{~h}-\mathrm{o})$ by using eqn. (12), it is necessary to formulate the volume of a single crystal, illustrated in Fig. 11 as a function of time. Here we will formulate the volume as functions of the crystallization time $(t)$ and the growth rate.

If a single crystal, illustrated in Fig. 11, is assumed to be a tetragonal prism with lateral size, $a \times a$ and thickness $l, \Phi$ is given by

$$
\Phi \propto a^{2} l
$$

We have shown previously ${ }^{6}$ and also in Fig. 7 and 8 that

$$
a=a^{*}+2 v t \text { and } l=l^{*}+2 u t
$$

Combination of eqn. (A1) and (A2) gives

$$
\Phi \propto\left(a^{*}+2 v t\right)^{2}\left(l^{*}+2 u t\right)
$$

In the case of an ECSC this can be approximated to

$$
\Phi \propto v^{2} u t^{3} ; \text { for } \Delta T(\mathrm{~h})>\text { a few } \mathrm{K}
$$

because $a$ and $l$ are much larger than $a^{*}$ and $l^{*}$ for a few K. It is shown that $v \propto u$ [see eqn. (4)], so eqn. (A4) becomes

$$
\Phi \propto u^{3} t^{3} ; \text { for } \Delta T(\mathrm{~h})>\mathrm{a} \text { few } \mathrm{K}
$$

As both $v$ and $u$ are functions of $\Delta T(\mathrm{~h}), \Phi$ is also a function of $\Delta T(\mathrm{~h})$, i.e.

$$
\Phi=\Phi[\Delta T(\mathrm{~h}), t]
$$

\section{References}

1 B. Wunderlich, Macromolecular Physics, Academic Press, New York, 1973, ch. 7 and 8.

2 H. Ohigashi, T. Watanabe, G. R. Li, T. Hattori and S. Takahashi, Jpn. J. Appl. Phys., 1990, 30, 111.

3 J. D. Hoffmann, G. T. Davis and J. I. Lauritzen Jr., Treatise on Solid State Chemistry, Plenum Press, New York, 1976, ch. 7, p. 580 .

4 D. M. Sadler, Nature (London), 1987, 326, 174.

5 M. Hikosaka, K. Amano, S. Rastogi and A. Keller, Crystallization of Polymers, NATO ASI Series, Kluwer, London, 1993, p. 331.

6 M. Hikosaka, K. Amano, S. Rastogi and A. Keller, Int. Polym. Phys. Symp., Tokyo, 1994, pp. 31-36.

7 S. Rastogi, M. Hikosaka, H. Kawabata and A. Keller, Macromolecules, 1991, 24, 6384.

8 M. Hikosaka, Polymer, 1987, 28, 1257; 1990, 31, 4.

9 M. Hikosaka, K. Tukijima, S. Rastogi and A. Keller, Polymer, $1992,33,2502$.

10 A. Keller, M. Hikosaka, S. Rastogi, A. Toda and P. J. Barham, J. Mater. Sci., 1994, 29, 2579.

11 F. P. Price, Nucleation, Marcel Dekker, New York, 1969, ch. 8

Paper 5/01401H; Received 7th March, 1995 